\title{
Osteogenic Activity of Locally Applied Small Molecule Drugs in a Rat Femur Defect Model
}

\author{
Jessica A. Cottrell, ${ }^{1,2}$ Francis M. Vales, ${ }^{1}$ Deborah Schachter, ${ }^{3}$ Scott Wadsworth, ${ }^{3}$ \\ Rama Gundlapalli, ${ }^{4}$ Rasesh Kapadia, ${ }^{5}$ and J. Patrick O'Connor ${ }^{1,2}$ \\ ${ }^{1}$ Department of Biochemistry \& Molecular Biology, New Jersey Medical School, University of Medicine and Dentistry of New Jersey, \\ Newark, NJ 07103, USA \\ ${ }^{2}$ Graduate School of Biomedical Sciences, New Jersey Medical School, University of Medicine and Dentistry of New Jersey, Newark, \\ NJ 07103, USA \\ ${ }^{3}$ Center for Biomaterials \& Advanced Technologies, Somerville, NJ 08876, USA \\ ${ }^{4}$ DePuy Orthopaedics, Inc., Warsaw, IN 46582, USA \\ ${ }^{5}$ Scanco USA, Inc., Wayne, PA 19087, USA \\ Correspondence should be addressed to J. Patrick O’Connor, oconnojp@umdnj.edu
}

Received 14 January 2010; Accepted 30 March 2010

Academic Editor: Anita M. Oberbauer

Copyright () 2010 Jessica A. Cottrell et al. This is an open access article distributed under the Creative Commons Attribution License, which permits unrestricted use, distribution, and reproduction in any medium, provided the original work is properly cited.

\begin{abstract}
The long-term success of arthroplastic joints is dependent on the stabilization of the implant within the skeletal site. Movement of the arthroplastic implant within the bone can stimulate osteolysis, and therefore methods which promote rigid fixation or bone growth are expected to enhance implant stability and the long-term success of joint arthroplasty. In the present study, we used a simple bilateral bone defect model to analyze the osteogenic activity of three small-molecule drug implants via microcomputerized tomography (micro-CT) and histomorphometry. In this study, we show that local delivery of alendronate, but not lovastatin or omeprazole, led to significant new bone formation at the defect site. Since alendronate impedes osteoclast-development, it is theorized that alendronate treatment results in a net increase in bone formation by preventing osteoclast mediated remodeling of the newly formed bone and upregulating osteoblasts.
\end{abstract}

\section{Introduction}

Bone remodeling is a delicate balance of two processes: bone deposition by osteoblasts and bone resorption by osteoclasts [1-3]. This equilibrium is regulated by local and systemic factors which influence the differentiation and activity of osteoblasts derived from mesenchymal cells and osteoclasts derived from hematopoietic precursors [4]. Manipulation of either of these processes can result in a net increase in bone formation. Moreover, modulation of these processes can be targeted to create therapeutic interventions to treat diseases associated with bone loss, such as osteoporosis, or to enhance arthroplasty success, where bone growth around the arthroplastic implant is needed to promote better fixation.

A critical factor for long-term success of arthroplastic joints is rigid stabilization of the implant within the skeletal site [5]. Osteolysis around arthroplastic implants leads to destabilization of the joint and necessitates revision surgery [69]. It is thought that movement of the arthroplastic implant within the bone can stimulate osteoclast activity or lead to abnormal implant wear, which in turn produces wear debris and an osteolytic inflammatory reaction [6]. Physical methods to promote rigid fixation of arthroplastic implants include press-fit implantation, screw augmentation, and cementing $[10,11]$. Other methods promote bone growth around the arthroplastic implant to promote rigid fixation, which include modifying the implant surface to promote osseous integration [12] and, more recently, coating the surface of the implant with an osteoconductive material, such as hydroxyapatite $[13,14]$. It is hypothesized that methods that would promote bone formation into or around an arthroplastic implant would enhance implant stability and increase the long-term success of joint arthroplasty. 
A potential method to enhance arthroplastic implant fixation would be use of pharmaceutical compounds that increase net bone formation into or around an arthroplastic implant. Such pharmaceutical compounds could be incorporated into the design of the implant or applied as an additional step during arthroplastic surgery. The pharmaceuticals could be bisphosphonates or proton pump inhibitors (PPIs) that interfere with osteoclast activity to increase bone density or statins that increase osteoblast activity. Nitrogen-containing bisphosphonates, such as alendronate, reduce osteoclastic resorption by specifically inhibiting farnesyl pyrophosphate synthase which disrupts key regulatory proteins and leads to osteoclast inactivity and apoptosis [15]. Diseases associated with excessive bone resorption, such as Paget's disease, myeloma, and osteoporosis, are currently treated with systemically delivered bisphosphonates [16]. Locally applied bisphosphonates also can inhibit bone resorption [17-19]. Osteoclasts resorb bone through the action of proton pumps that acidify the extracellular environment within the ruffled border zone [15]. Thus inhibiting the activity of proton pumps may also impair osteoclast activity. Omeprazole is a gastric PPI currently used to treat acid-related diseases such as peptic ulcer diseases and gastroesophageal reflux [20]. This drug inhibits various protein pumps including the vacuolar-type $\mathrm{H}(+)$-ATPase which is important in the formation of the acidic environment in the extracellular ruffled border zone of osteoclasts [21-25]. Statins, like lovastatin, inhibit HMG-CoA reductase and are thought to shunt uncommitted osteoprogenitor cells in the marrow to osteoblastic differentiation leading to a positive effect on bone formation [26]. Research has shown that statins can increase bone formation in vitro and in vivo when given locally or in very large doses systemically $[27,28]$. Statins have also been shown to increase the expression of BMP-2 which can positively affect bone formation $[29,30]$.

As a first step to developing new pharmaceutical enhancements for arthroplastic implant fixation, the present study examined whether local application of a bisphosphonate, (alendronate), a PPI (omeprazole), or a statin (lovastatin) could increase local bone formation in a cortical bone defect model.

\section{Materials and Methods}

2.1. Hydrogel Implant Preparation. All blanks and drugladen implants were produced at Ethicon (Somerville, NJ). For blank implants, sodium hyaluronate powder (Novamatrix, Pharma 150) was weighed (2.0 grams) and transferred to the large mixing bowl of a Caleva Mixer Torque Rheometer (MTR) while mixer paddles were turning at $50 \mathrm{rpm}$. A 1:1 mixture of ethanol and distilled water was prepared and $3 \mathrm{ml}$ of the solution was added to the mixing powder in $0.5 \mathrm{ml}$ aliquots. Wet granulation was removed from large mixing bowl and the Caleva MTR was refitted with the extruder accessory. The wet granulation was placed in the hopper of the extruder with a single screw turning at $50 \mathrm{rpm}$. A die with a configuration of $3 \mathrm{~mm}$ holes was placed at the end of the extruder screw and wet granulation was extruded into 3 strands of $3 \mathrm{~mm}$ in diameter. Strands were immediately cut into $3 \mathrm{~mm}$ pellet lengths and placed in a vacuum oven for overnight drying. The average weight of implants was $10.0 \pm$ $1.4 \mathrm{mg}$.

For each drug-laden implant, alendronate, lovastatin, or omeprazole (Sigma, St. Louis, MO) was weighed and dissolved in $4 \mathrm{ml}$ of a 1:1 ethanol water solution and added to 2.5 grams sodium hyaluronate in $0.5 \mathrm{ml}$ aliquots as described above. At the higher concentration of alendronate, $5.75 \mathrm{mg}$ of active compound was dispersed in 2.5 grams of sodium hyaluronate $(0.23 \% \mathrm{w} / \mathrm{w})$. At the lower concentration $1.35 \mathrm{mg}$ of alendronate was dispersed in 2.5 grams of sodium hyaluronate $(0.05 \% \mathrm{w} / \mathrm{w})$. The average mass of the higher and lower concentration pellets was $11.4 \pm 0.6$ and $13.6 \pm 0.8 \mathrm{mg}$, respectively. At the higher concentration of lovastatin, $11.56 \mathrm{mg}$ of active compound was dispersed in 2.5 grams of sodium hyaluronate $(0.5 \% \mathrm{w} / \mathrm{w})$. At the lower concentration $1.3 \mathrm{mg}$ of lovastatin was dispersed in 2.5 grams of sodium hyaluronate $(0.05 \% \mathrm{w} / \mathrm{w})$. The average mass of the higher and lower concentration pellets was $13.0 \pm 0.7$ and $16.1 \pm 0.5 \mathrm{mg}$, respectively. At the higher concentration of omeprazole, $26.71 \mathrm{mg}$ of active compound was dispersed in 2.5 grams of sodium hyaluronate $(1 \% \mathrm{w} / \mathrm{w})$. At the lower concentration $2.71 \mathrm{mg}$ of omeprazole was dispersed in 2.5 grams of sodium hyaluronate $(0.1 \% \mathrm{w} / \mathrm{w})$. The average mass of the higher and lower concentration pellets was $13.7 \pm$ 0.6 and $14.6 \pm 0.5 \mathrm{mg}$, respectively. The concentration of the active compound within drug-laden implants was confirmed by HPLC analysis.

2.2. Coating of Pellets. Poly (lactide co-glycolide) (PLGA) was dissolved in ethyl acetate to the target concentration. The pellets were individually placed in respective $1.5 \mathrm{ml}$ siliconized polypropylene microcentrifuge tubes. Five pellets were placed on a teflon substrate. A pipette was used to coat the pellet with the coating solution. The volume of each coating application was $50 \mathrm{ul}$. Pellets were vortexed in the closed microcentrifuge tube in order to more uniformly coat the pellet. Pellets were dried in a ventilated hood for 2-3 hours followed by drying overnight in a vacuum oven at room temperature. Afterwards, the exterior bottom of the microcentrifuge tube was dipped quickly in liquid nitrogen to dislodge the pellet from the tube.

2.3. Animal Procedures. Male Sprague-Dawley rats that weighed $544 \pm 52$ grams at the time of surgery and 555 \pm 56 grams when euthanized 3 weeks later were used. The rats were housed in pairs, maintained in a 12-hour light dark cycle, and provided food and water ad libitum. All animal procedures were approved by the UMDNJ-New Jersey Medical School Institutional Animal Care and Use Committee prior to initiation of the project.

Bilateral defects were made in the distal femur of each rat to assess local drug effects on bone formation. Rats were anesthetized by intraperitoneal injection of ketamine $(50 \mathrm{mg} / \mathrm{kg})$ and xylazine $(10 \mathrm{mg} / \mathrm{kg})$. Hind limbs were prepared for surgery by shaving the knee areas and scrubbing with betadine. A lateral $1 \mathrm{~cm}$ incision was created above the knee on each thigh. The overlying muscle was bluntly dissected to the surface of the distal femur. Using a $3 \mathrm{~mm}$ 
diameter burr and a variable speed drill (Dremel, Racine, WI), a defect in the lateral aspect of each femur was created through the cortical bone to expose the marrow cavity [31]. Each defect site was irrigated with saline and sponged dry with gauze. A dry hydrogel implant was inserted into the defect with the aid of a $1 \mathrm{cc}$ tuberculin syringe (without needle) moistened at the tip with saline. The wounds were closed in two layers with resorbable sutures. Radiographs of each animal were made to confirm and record the initial defect.

Seven treatment groups were tested: (a) empty defect, (b) low-dose alendronate ( $7 \mathrm{ug} / \mathrm{implant}$ ), (c) high-dose alendronate (26 ug/implant), (d) low-dose lovastatin (8 ug/implant), (e) high-dose lovastatin (65 ug/implant), (f) low-dose omeprazole (15 ug/implant), and (g) high-dose omeprazole (137 ug/implant). Each rat received a placebo hydrogel implant (blank) and one of the above treatments. Treatments were randomized to each animal, and implantation of the blank was randomized to the left or right femur. In order to determine if drug treatment enhanced healing, rats were euthanized when the blank implant filled defects appeared to be undergoing healing but were not yet fully healed. Based on a pilot study, burr defects filled with blank implants were actively healing at 3 weeks but not fully healed. Therefore, six to eight rats were used per treatment group and all rats were euthanized and femurs analyzed at 3 weeks post surgery.

2.4. Micro-Computed Tomography. Resected femurs were cleaned of soft tissue without disturbing the surgical site and photographed to record any gross, macroscopic findings. The distal portion of each femur containing the defect site was removed and fixed in buffered formalin. After 47 days of fixation, the femurs were washed in 2 overnight changes of $70 \%$ ethanol prior to microcomputerized tomography (microCT) analysis (SCANCO, Wayne, PA). Threedimensional $\mu \mathrm{CT}$ images were made to analyze the bone mineral density (BMD) and bone volume (BV) of the defect site. The specimens were scanned in $70 \%$ ethanol to prevent drying. All samples were scanned on a Scanco Medical $\mu \mathrm{CT}$ 40 system at an energy of $55 \mathrm{Kvp}$ and intensity of $145 \mu \mathrm{A}$ with a voxel size of 16 microns isotropic using a $0.5 \mathrm{~mm}$ Al filter. Cone-Bean reconstruction was performed. All scan and reconstruction parameters applied were identical for all specimens. The data was analyzed using the Scanco Analysis Software bundled with the microCT system. The scanner acquisition and software had built-in image intensity to density conversion capability which enabled quantitative analysis of BMD in units of HA mg/cc. The region of interest (ROI) was specified as fixed distance of $4.4 \mathrm{~mm}$ (275 slices per specimen) and was consistent between samples. In this area BMD and BV were determined. BMD is defined as the amount of bone mineral per unit volume of bone tissue $\left(\mathrm{g} / \mathrm{cm}^{3}\right)$. In addition, $\mathrm{BV}\left(\mathrm{mm}^{3}\right)$ was expressed as a percentage of the total ROI volume.

2.5. Histological Procedures. Following microCT scanning, samples were embedded in polymethylmethacrylate (PMMA) following standard procedures [32]. A single section was cut through the defect site perpendicular to the long axis of the femur. Sections were polished and stained with van Gieson's picrofuchsin to stain mineralized tissue red and Stevenel's blue to stain cartilage deep blue [33-35]. The amount of new bone measured within the defect (defined as all bone within ROI-1 in Figure 4(a)-left) and on the periphery (defined as all bone outside of ROI-2 in Figure 4(b)-right) was measured by histomorphometric analysis of digital images using Image Pro software (version 5.0, Media Cybernetics, Inc., Bethesda, MD).

2.6. Data Analysis. Data were compared between the blank values of the empty defect group and the blank values of the treatment groups using an analysis of variance (ANOVA). This comparison determined whether any treatment had a systemic effect. Local osteogenic effects were identified by comparing experimental and blank pellets using a t-test within a treatment group.

\section{Results}

3.1. Drug Release and Elution Analysis. In vitro release of omeprazole and lovastatin from the implants was measured by HPLC (data not shown). Alendronate could not be detected using UV or visible light spectrometry and therefore was not analyzed using this method. Instead the in vitro release of alendronate was measured by liquid chromatography -mass spectrometry. The data demonstrated that a burst of drug was released on day one which declined by day three. This trend was observed regardless of the drug, concentration of drug (high or low dose levels), or percent PLGA coating. Overall, these studies showed that drug release from implants did not occur by simple diffusion from the hyaluronate pellets. Instead there appeared to be a strong drug-polymer interaction which made drug release, following the initial burst release, dependent on degradation of the implant. Since coating the implants with PLGA had no impact on drug release rate, the implants used in this study were left uncoated.

3.2. Observations regarding the Surgical Procedure. The surgical procedure was easily accomplished and well tolerated by the rats. Implantation of the hydrogel-drug implants was accomplished by touching one end of the dry implant with the end of a $1 \mathrm{cc}$ tuberculin syringe (without needle) that had been dipped in saline. The implant "stuck" to the end of the syringe and allowed it to be easily placed into the defect. It was noted that the heavier implants were more difficult to implant into the defect which was likely related to the increased diameter of the implant.

3.3. Disposition of Animals and Femur Samples. The experimental design and loss of animals in the study are summarized in Table 1. One anticipated complication was postsurgical fracture which occurred in 3 of the 90 femurs used in the study. Two fractures occurred in the alendronate treatment groups and one in a control blank femur of the omeprazole treatment group. Only one of the 90 femurs developed an infection. Two rats died from the surgical anesthesia after the surgical procedure was complete, that is, the animals never regained consciousness. Finally, femurs 
TABle 1: Summary of Animal Disposition.

\begin{tabular}{|c|c|c|c|c|c|c|c|c|}
\hline \multicolumn{9}{|c|}{ Postsurgical } \\
\hline \multirow[t]{2}{*}{ Treatment versus Blank } & \multirow[t]{2}{*}{ Initial Group Size } & \multicolumn{4}{|c|}{ Morbidity \& Mortality } & \multirow[t]{2}{*}{ Histology Error } & \multicolumn{2}{|c|}{ Group Size for Each Analysis } \\
\hline & & Fracture & Infection & Anesthesia & Other & & microCT & Histomorph. \\
\hline Empty Defect & 6 & 0 & 0 & 0 & 1 & 0 & 5 & 5 \\
\hline Low-Dose Alendronate & 6 & $1(\mathrm{Rx})$ & 1 & 0 & 0 & 1 & 4 & 3 \\
\hline High-Dose Alendronate & 6 & $1(\mathrm{Rx})$ & 0 & 0 & 0 & 0 & 5 & 5 \\
\hline Low-Dose Lovastatin & 6 & 0 & 0 & 0 & 0 & 0 & 6 & 6 \\
\hline High-Dose Lovastatin & 7 & 0 & 0 & 1 & 0 & 0 & 6 & 6 \\
\hline Low-Dose Omeprazole & 6 & 0 & 0 & 0 & 0 & 0 & 6 & 6 \\
\hline High-Dose Omeprazole & 8 & $1(\mathrm{Bl})$ & 0 & 1 & 0 & 1 & 6 & 5 \\
\hline Totals & 45 & 3 & 1 & 2 & 1 & 2 & 38 & 35 \\
\hline
\end{tabular}

from two rats were not used because the defect could not be located after resection of the femur. Immediate postoperative radiographs confirmed that the rats indeed had bilateral femur defects. Yet for two femurs, no defect could be observed after 3 weeks of healing suggesting that the defect had completely healed. One of the completely healed defects was a blank of the high-dose omeprazole group and the other was a blank defect in the low-dose alendronate group.

3.4. Radiographic Observations of the Healing Defects. Radiographs were made for each animal immediately after surgery and again at 3 weeks (Figure 1). Defects were plainly evident in most radiographs. Visual evidence of healing also was apparent in most of the radiographs. However, the radiographs appeared to present a spectrum of healing rather than having distinct classes of healed (Figure 1(c)) versus not healed (Figure 1(b)). Beyond using these radiographs to identify postsurgical complications, such as fractures or infections, the radiographs were not scored or in any other way correlated to the experimental treatments.

3.5. Microcomputerized Tomography (microCT) Analysis of the Healing Defects. The primary outcome measure was microCT quantification of new bone at the defect site for each femur. In addition, bone mineral density of the newly formed bone also was measured. Femurs were scanned in a blinded fashion in that the operator was unaware of the treatments used in each femur. Representative 3D reconstructions of an empty defect, blank-treated defect, and defects from the high-dose drug treatment groups are shown in Figure 2. The experimental design enabled a direct comparison of new bone formation by comparing femur defects treated with drug (or left empty) with its contralateral femur defect treated with a blank. Treatment and blank defect new bone volumes were compared with $t$-tests (Table 2 and Figure 3 ). The data indicate that only alendronate treatment, either low or high dose, led to significantly more bone formation at the defect site. Analysis of variance (ANOVA) and post hoc Holm-Sidak tests of bone formation found that only the alendronate groups were significantly different than all other treatment groups $(P<.001$ and $P=.003)$ but that

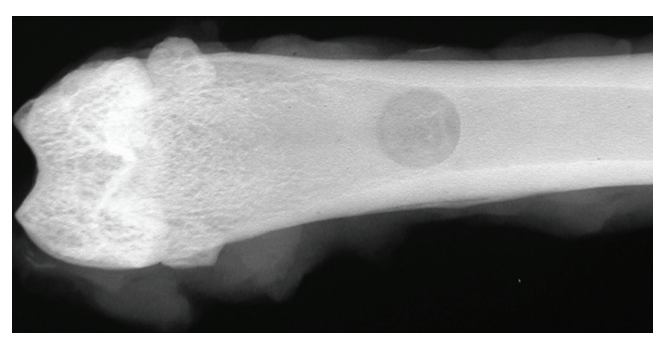

(a)

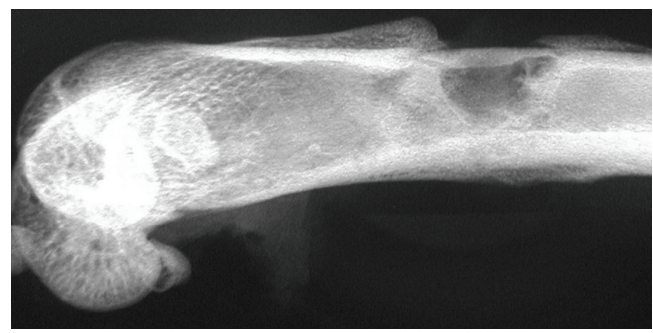

(b)

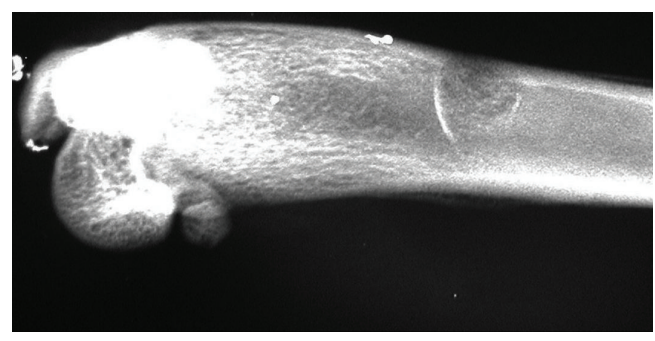

(c)

Figure 1: Post-Surgery Radiographs. Shown are radiographs of distal femur defects filled with placebo hydrogel inserts (blanks) immediate after surgery (a) or 3 weeks later (b-c). The radiographs revealed a range of healing from partially healed (b), to fully healed (c). Periosteal bone formation was evident in many samples (b).

no difference was found between the low- and high-dose alendronate groups $(P=.300)$.

There were statistically significant differences between the blank defect bone volumes of the different treatment groups (ANOVA $P=.043$ ), indicating that locally 

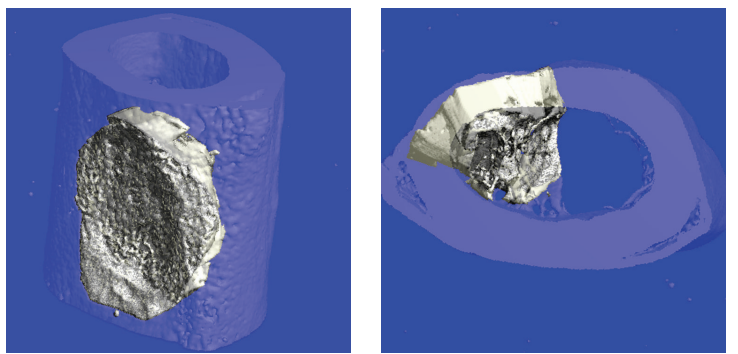

(a)
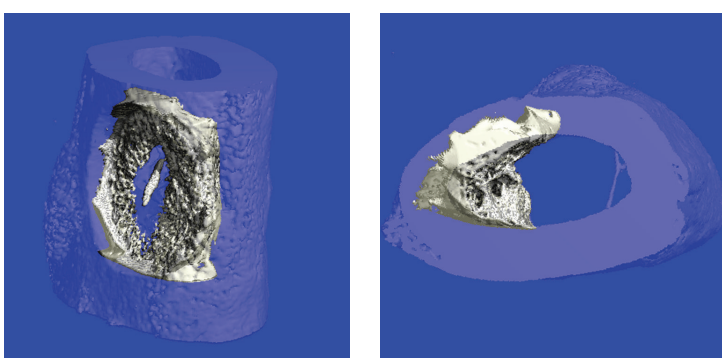

(b)
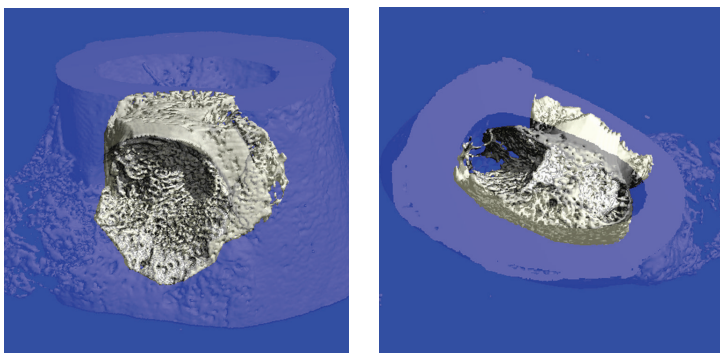

(c)
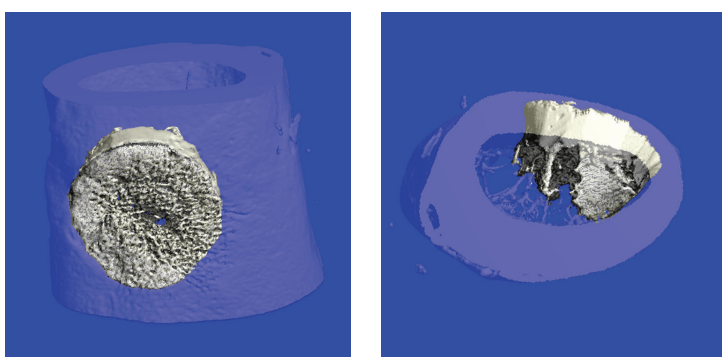

(d)
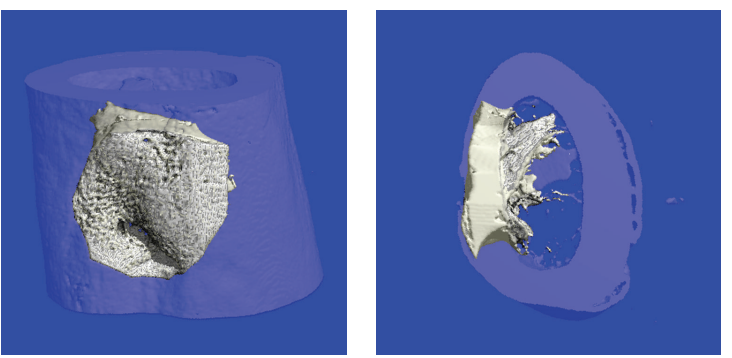

(e)

Figure 2: MicroCT Reconstructions of Femur Defects. Femurs were harvested 3 weeks after surgery and analyzed for bone formation by microCT. Shown are lateral (left column) and cross-sectional projections (right column) of (a) an empty defect and defects treated with (b) a blank, (c) high-dose alendronate, (d) high-dose lovastatin, and (e) high-dose omeprazole.

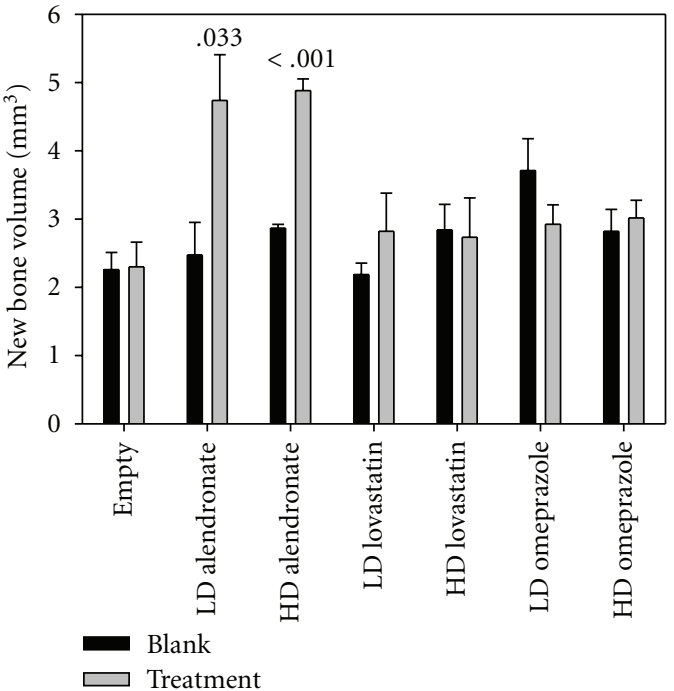

Figure 3: New Bone Volume Quantified from the MicroCT Analysis $\left(\mathrm{mm}^{3}\right)$. The volume of new bone $\left(\mathrm{mm}^{3}\right)$ formed in each treatment group (gray bars) was compared to the volume of new bone formed in the contralateral, blank-treated femurs (black bars) for that group using Students $t$-tests. Significant $P$-values are shown (LD: low-dose; HD: high-dose).

administered omeprazole had a systemic effect on bone formation. New bone volume in the blank-treated femurs of the low-dose omeprazole treatment group was significantly greater than that from the blank-treated empty defect group $(P=.004)$, low-dose lovastatin group $(P=.002)$, and lowdose alendronate group $(P=.020)$. No difference in new bone mineral density was detected between treatment groups (Table 3).

3.6. Histomorphometric Analysis of the Healing Defects. Representative histological sections from an empty defect and each high-dose treatment group are shown in Figure 4. New bone was evident in all the samples but little or no cartilage was visible. New bone area was measured for each sample and was classified as being either within the defect and intramedullary canal (defect site, Figure 4(a)-left, shown as area within ROI-1) or outside the defect (peripheral, Figure 4(b)-right, shown as the area outside of ROI-2). Significantly more, total (defect site and peripheral), defect site, and peripheral new bone was measured in the lowdose alendronate-treated samples as compared to the corresponding blank samples $(P=.004,0.020$, and .006, resp.). In addition, significantly more bone was measured in the defect site of the high-dose alendronate samples $(P=.044)$. Though the total and peripheral new bone areas were higher in the high-dose alendronate group, these values were not significantly higher than the contralateral blank defect values (Figure 5). No other significant effects were noted.

\section{Discussion}

In the present study, local release of antiresorptive and osteogenic drugs from hydrogel implants was used as an 
TABLE 2: Effects of Drug Therapy on New Bone Volume.

\begin{tabular}{|c|c|c|c|c|c|c|c|}
\hline \multirow{2}{*}{ Treatment } & \multirow{2}{*}{$\begin{array}{c}\text { Implant } \\
\text { weight }(\mathrm{mg})\end{array}$} & \multirow{2}{*}{$\begin{array}{c}\text { Percent } \\
\text { drug }(w / w)\end{array}$} & \multirow{2}{*}{$\begin{array}{c}\text { Approx. } \\
\text { site dose (ug) }\end{array}$} & \multirow{2}{*}{ Group size } & \multicolumn{2}{|c|}{ Bone volume $\left(\mathrm{mm}^{3}\right.$; mean \pm S.D. $)$} & \multirow{2}{*}{$\begin{array}{c}t \text {-test } \\
P \text { value }\end{array}$} \\
\hline & & & & & Drug & Blank & \\
\hline Empty Defect & 0 & 0 & 0 & 5 & $2.312 \pm 0.810$ & $2.271 \pm 0.564$ & .929 \\
\hline Low-Dose Alendronate & $13.6 \pm 0.8$ & 0.05 & 6.8 & 4 & $4.749 \pm 1.343$ & $2.487 \pm 0.952$ & .033 \\
\hline High-Dose Alendronate & $11.4 \pm 0.6$ & 0.23 & 26.2 & 5 & $4.893 \pm 0.385$ & $2.897 \pm 0.125$ & $<.001$ \\
\hline Low-Dose Lovastatin & $16.1 \pm 0.5$ & 0.05 & 8.0 & 6 & $2.833 \pm 1.370$ & $2.199 \pm 0.409$ & .303 \\
\hline High-Dose Lovastatin & $13.0 \pm 0.7$ & 0.5 & 65 & 6 & $2.746 \pm 1.410$ & $2.853 \pm 0.916$ & .880 \\
\hline Low-Dose Omeprazole & $14.6 \pm 0.5$ & 0.1 & 14.6 & 6 & $2.936 \pm 0.695$ & $3.722 \pm 1.145$ & .181 \\
\hline High-Dose Omeprazole & $13.7 \pm 0.6$ & 1 & 137 & 6 & $3.029 \pm 0.634$ & $2.833 \pm 0.783$ & .645 \\
\hline
\end{tabular}

TAble 3: Effects of Drug Therapy on New Bone Density (mg/cc HA).

\begin{tabular}{lcccc}
\hline Treatment versus Blank & Group Size & \multicolumn{2}{c}{ Bone density (mg/cc HA; mean \pm S.D. } & Blank \\
\hline Empty Defect & 5 & $998 \pm 52$ & $1023 \pm 36$ & 0.401 \\
Low-Dose Alendronate & 4 & $966 \pm 20$ & $988 \pm 32$ & 0.284 \\
High-Dose Alendronate & 5 & $975 \pm 42$ & $1012 \pm 32$ & 0.156 \\
Low-Dose Lovastatin & 6 & $1027 \pm 49$ & $993 \pm 16$ & 0.138 \\
High-Dose Lovastatin & 6 & $1018 \pm 20$ & $1016 \pm 45$ & 0.200 \\
Low-Dose Omperazole & 6 & $1003 \pm 22$ & $1009 \pm 26$ & 0.487 \\
High-Dose Omperazole & 6 & & & 0.673 \\
\hline
\end{tabular}

approach to enhance bone formation in a cortical defect as a preliminary test model for increasing bone formation around arthroplastic implants. The data demonstrated that local treatment with the bisphosphonate alendronate led to significantly more new bone at the rat femur defect site as compared to the controls (both blank and empty defect) and the other osteogenic drug treatments. Comparison of total new bone formation at the defect site between treatment groups found that both doses of alendronate led to significantly more bone formation as compared to omeprazole or lovastatin.

The paired design of this study controlled for biological differences between rats. With this method of local drug administration, a risk existed for systemic distribution of the agents that could influence the bone on the control (blank) side. However, a separate control group (empty defect-blank implant) was completed so that we could analyze the local effects as well as the systemic effects of each drug. MicroCT analysis of bone volume detected a positive systemic effect in the control side of the low-dose omeprazole group. However, no local effect on bone healing was found with omeprazole treatment. These results suggest that higher concentrations of omeprazole (such as those applied locally or that circulate systemically in the high-dose omeprazole group) do not increase bone formation while a low concentration (similar to the concentration circulating systemically in the low-dose omeprazole group) can increase bone formation. Additional studies are needed to confirm this apparent omeprazole effect. No systemic effect was detected for any other treatment groups.
We suspect that the surgical insult produces a significant bone regenerative response in the rats. This response likely includes a significant activation of periosteal osteoblasts and bone marrow preosteoblasts to form new bone. However, this flux of osteoblasts would have contributed to bone formation in all test groups and does not explain the approximate 2 -fold increase in bone volume found in rats treated with the alendronate hydrogel implants.

The mechanism by which alendronate increased bone formation was not investigated in these studies. Alendronate is expected to prevent osteoclast mediated remodeling of the newly formed bone, which could in part account for the increased amount of mineralized tissue measured in the alendronate treatment groups [36, 37]. Alendronate and other nitrogen-containing bisphosphonates also can increase osteoblast proliferation [38-40], prevent osteoblast apoptosis [41, 42], inhibit differentiation of mesenchymal stem cells into adipocytes $[43,44]$, and promote osteoblast differentiation and activity including enhancing expression of Runx2 and BMP-2 [39, 40, 45, 46]. Alendronate effects on osteoblasts appear to be mediated through ERK and JNK activation and alteration of the mevalonate pathway $[38,41,42,44,47]$. Thus, alendronate can have both anabolic and anticatabolic effects on bone.

We suggest that local alendronate treatment enhanced bone formation and reduced osteoclast-mediated remodeling at the cortical defect site leading to a large increase in bone formation. Our results are similar to those of Jakobsen et al. who demonstrate that local alendronate treatment increased fixation of implants inserted in cancellous bone 

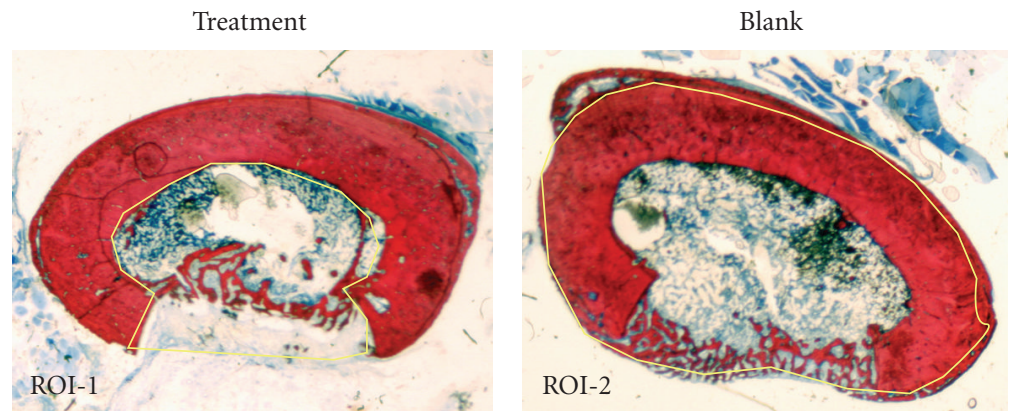

(a)
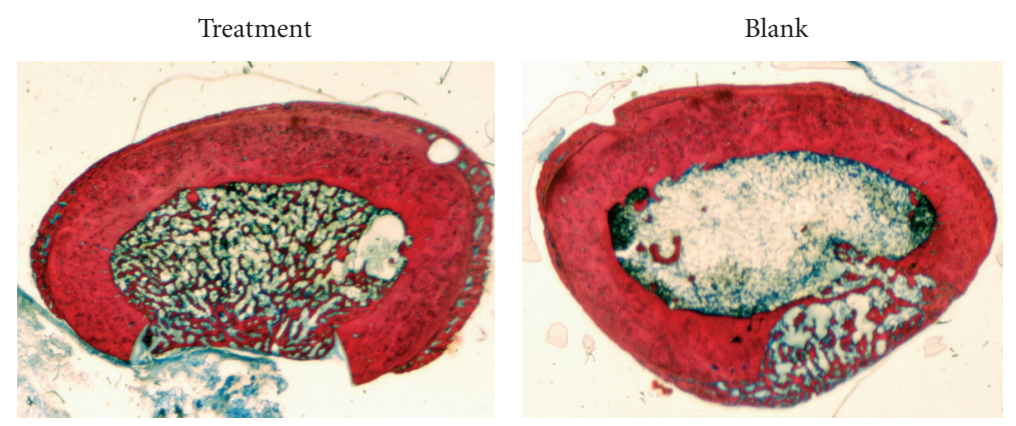

(b)
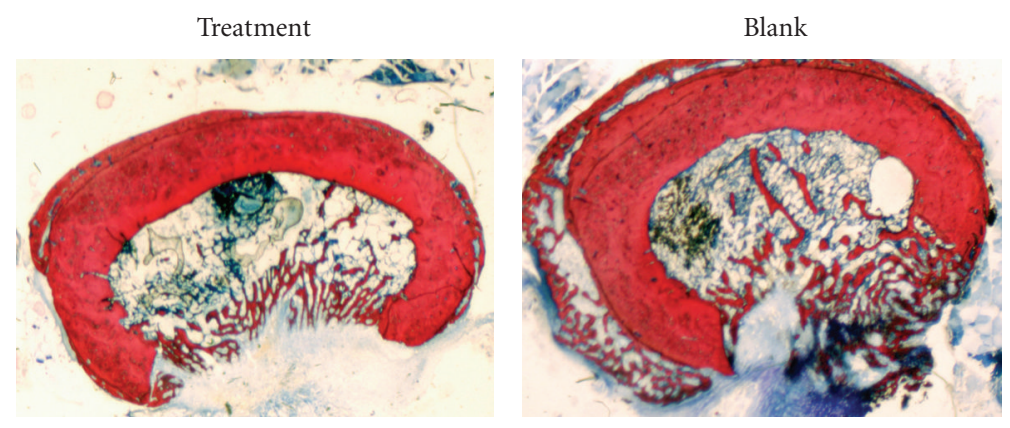

(c)
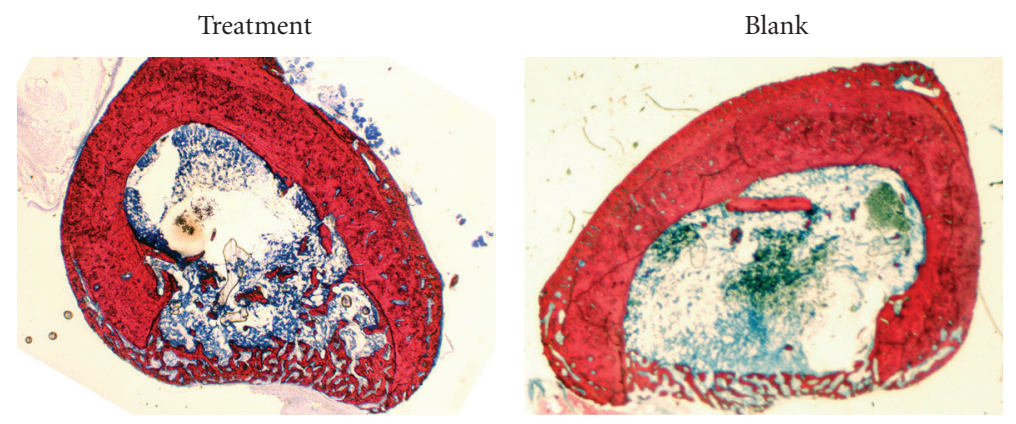

(d)

FIGURE 4: Histological Sections of Femur Defects. Following micro CT evaluation, specimens were plastic embedded, sectioned through the defect, and stained for mineralized tissue (red) and cartilage (deep blue). Shown are representative cross-sections from (a) an empty defect and defects treated with (b) high-dose alendronate, (c) high-dose lovastatin, and (d) high-dose omeprazole. ROI-1 is shown and included the defect site and marrow space (a)-left. ROI-2 is shown and bone outside of ROI-2 was considered peripheral bone formation (a)-right. Corresponding blank-treated defects from each treatment group are shown in the right column. Mineralized tissue was evident in all defect sites but little or no cartilage was observed. 


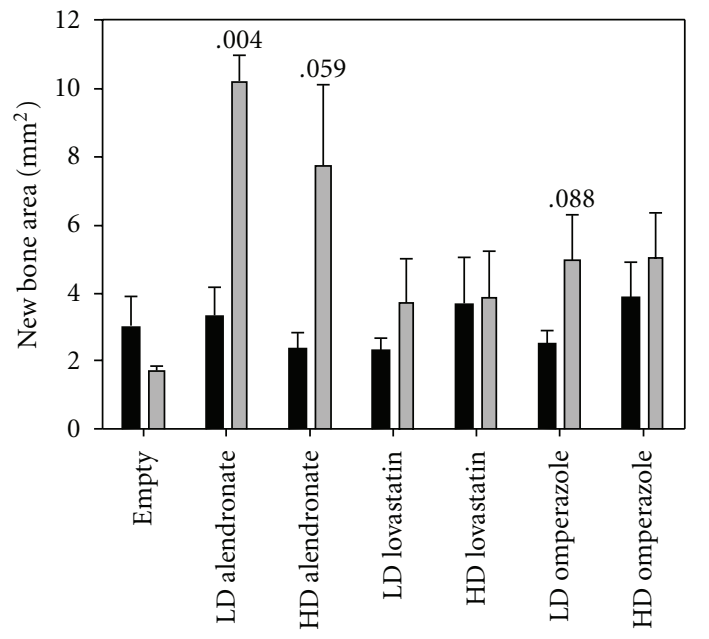

(a)

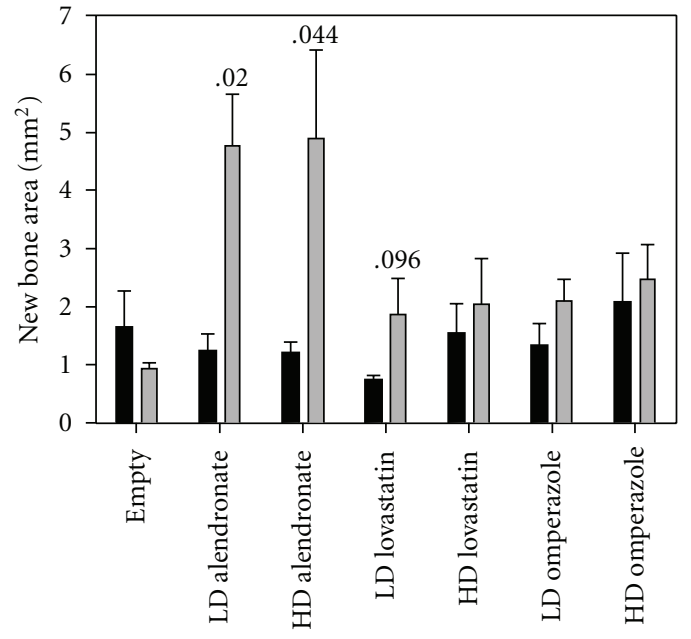

(b)

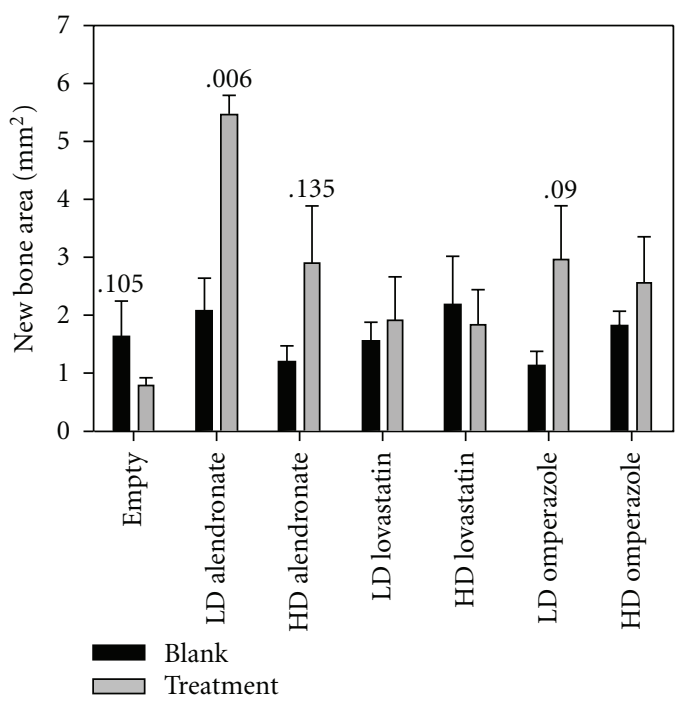

(c)

Figure 5: Histomorphometric Analysis of Bone Formation. Areas of new bone formation were measured from cross-sections of each femur defect. The total new bone area (a) was further categorized as being within the defect site and marrow space (b) or on the femur periphery (c). New bone area for each treatment group (gray bars) was compared to the new bone area in the contralateral, blank-treated femurs (black bars) for that group using Students $t$-tests. Significant $P$ values are shown (low-dose, LD; high-dose, HD).

after 4 and 12 weeks in a canine model by increasing bone volume and density [17, 18]. These results contrast a rabbit femoral condyle study where alendronate had no positive effect when it was incorporated into bone cement and inserted into the defect. However, in this rabbit study, alendronate appeared to evoke a toxic response which may have contributed to the absence of a positive bone healing response [15]. This suggests that alendronate effect on osteoblast activity may be more important for increasing bone formation during healing of bone defects than alendronate effects on osteoclast activity. Future studies to characterize the cell types and quantify osteoblast- and osteoclast-related factors at the bone defect site should provide a better understanding of how these drugs alter the local bone forming environment.
Since an overall aim of adjuvant therapies for arthroplasty is to increase early fixation of these implants and reduce instability $[18,48]$, local alendronate treatment could increase the long-term success of arthroplastic surgeries by preventing osteolysis and promoting osteogenesis around the implant [49-51]. Treatment with lovastatin showed no positive effects on bone formation at the implant site. Lovastatin is expected to promote osteoblast activity by inducing BMP2 expression but would not directly affect osteoclast activity [28]. Previous studies have shown that large doses of orally administered lovastatin can stimulate bone formation [28]. If lovastatin did induce a large bone formation response in this study, it is possible that subsequent osteoclast mediated remodeling may have destroyed most of the initial bone. The data indicate that this hypothesis is unlikely 
since bone remodeling would have likely led to increased bone mineral density and no such increase was observed (Table 3). Lovastatin concentration may have contributed to the lack of bone formation. The highest concentration of lovastatin incorporated per implant was approximately $0.01 \mathrm{mg} / \mathrm{kg}$ body weight. This is similar to the dose used by Gutierrez et al. which demonstrated that 5 consecutive days of transdermally applied lovastatin $(0.01 \mathrm{mg} / \mathrm{kg}$ per day $)$ to ovariectomized rats increased trabecular bone volume by $30-60 \%$ as well as increasing bone formation [27]. However, based on the in vitro elution studies, the largest portion of the lovastatin dose in this model would have been released by day 1 (which is expected to be less than $0.01 \mathrm{mg} / \mathrm{kg}$ ) with subsequent smaller daily releases of drug. Therefore, lovastatin concentration or length of release may have contributed to the absence of increased bone formation.

Experimentally, omeprazole produced no consistent effect on bone formation. Omeprazole is expected to inhibit proton pumps $(\mathrm{H}+\mathrm{K}+\mathrm{ATPase})$ in osteoclasts and prevent bone resorption [52]. The large amount of new bone volume in the blank-treated defects of the low-dose omeprazole treatment group as compared to the blank-treated defects from the empty $(P=.004)$, low-dose alendronate $(P=.020)$, and low-dose lovastatin $(P=.002)$ groups was unexpected (see Table 2 and Figure 3 ). This elevation suggests a systemic effect of low-dose omeprazole, but a similar effect was not found in the high-dose omeprazole. One plausible explanation is that the local omeprazole concentrations as well as the systemic levels of omeprazole in the high-dose group were too high to promote formation. If this is true, lower doses of omeprazole may help promote bone formation. This hypothesis will require additional investigation.

Alendronate treatment was superior to lovastatin or omeprazole in this in vivo assay of bone formation. Peter et al. found that local zoledronate (another bisphosphonate) doses of 0.2 and $2.0 \mathrm{ug}$ were not effective in promoting osteogenesis while doses of $8.5 \mathrm{ug}$ and higher (maximum tested was $16 \mathrm{ug}$ ) all stimulated osteogenesis [53]. In contrast, Bodde et al. found that a local alendronate dose of $8.2 \mathrm{mg}$ inhibited bone formation in a rabbit femoral condyle defect model [15]. Consequently we tested alendronate using 6.8 and 26.2 ug doses to identify any potential dose response effects in this dose range or any potential negative effect of the drug. Similar to Peter et al. [53], we found that both bisphosphonate doses increased bone formation at the defect site. Nominally, bisphosphonates can be incorporated into bone, and since the defect site is actively producing new bone, it is likely that release of alendronate from the implant led to a locally high concentration of drug that remained at the site. In contrast, lovastatin and omeprazole are not known to be incorporated into the bone matrix. Thus the effective release kinetics between alendronate, lovastatin, and omeprazole are likely very different. In addition, preliminary in vitro studies indicated a sharp burst of drug release initially from the pellets which dropped off precipitously (within three days). Therefore, additional experiments to test implants that show a longer-lasting release of lovastatin or omeprazole may show that these compounds also are effective osteogenic compounds.

This study screened 3 drugs for potential use as agents to enhance local bone formation. As such, the study has significant limitations. First, the study design used only a single time point, which may not have captured early or later positive effects of the drugs. There is also the concern whether results from rodent models will translate to similar effects in humans. The rat femur lacks large amounts of trabecular bone that is typically found in the metaphyses of human long bones. Consequently, the drugs in this study appeared to primarily affect cortical bone rather than trabecular bone as would be expected in humans undergoing an arthroplastic procedure. The bilateral experimental design controlled for potential drug systemic effects; however, systemic and local drug levels were not measured in vivo. Drug release data would be helpful in designing future studies and for comparing drug effects when released from different carriers or when used in different models.

\section{Conclusions}

Our data demonstrates that treatment with alendronate led to a significant increase in defect site bone volume while omeprazole or lovastatin treatment had no significant effect on bone formation in this model using the hydrogel carrier. Local alendronate treatment may be useful in increasing the outcome success of arthroplastic surgeries by preventing osteolysis around the skeletal surgical site. Further analysis of lovastatin and omeprazole would be necessary to determine their efficacy at promoting bone formation in this model.

\section{References}

[1] T. Katagiri and N. Takahashi, "Regulatory mechanisms of osteoblast and osteoclast differentiation," Oral Diseases, vol. 8, no. 3, pp. 147-159, 2002.

[2] T. J. Martin and N. A. Sims, "Osteoclast-derived activity in the coupling of bone formation to resorption," Trends in Molecular Medicine, vol. 11, no. 2, pp. 76-81, 2005.

[3] J. P. Stains and R. Civitelli, "Cell-to-cell interactions in bone," Biochemical and Biophysical Research Communications, vol. 328, no. 3, pp. 721-727, 2005.

[4] L. G. Raisz, "Physiology and pathophysiology of bone remodeling," Clinical Chemistry, vol. 45, no. 8, pp. 1353-1358, 1999.

[5] T. Otani and L. A. Whiteside, "Failure of cementless fixation of the femoral component in total hip arthroplasty," Orthopedic Clinics of North America, vol. 23, no. 2, pp. 335-346, 1992.

[6] G. Holt, C. Murnaghan, J. Reilly, and R. M. D. Meek, "The biology of aseptic osteolysis," Clinical Orthopaedics and Related Research, vol. 460, pp. 240-252, 2007.

[7] K. Lee and S. B. Goodman, "Current state and future of joint replacements in the hip and knee," Expert Review of Medical Devices, vol. 5, no. 3, pp. 383-393, 2008.

[8] S. B. Goodman, "Wear particles, periprosthetic osteolysis and the immune system," Biomaterials, vol. 28, no. 34, pp. 5044$5048,2007$. 
[9] S. B. Goodman, K. J. Oh, S. Imrie, K. Hwang, and M. Shegog, "Revision total hip arthroplasty in juvenile chronic arthritis: 17 revisions in 11 patients followed for 4-12 years," Acta Orthopaedica, vol. 77, no. 2, pp. 242-250, 2006.

[10] S. Abdulghani, J. S. Wang, I. McCarthy, and G. Flivik, "The influence of initial pressurization and cup introduction time on the depth of cement penetration in an acetabular model," Acta Orthopaedica, vol. 78, no. 3, pp. 333-339, 2007.

[11] N. A. Munro, M. Nicol, S. Selvaraj, S. M. Hussain, and D. F. Finlayson, "Femoral cement pressurization in hip arthroplasty: a comparison of 3 systems," Journal of Arthroplasty, vol. 22, no. 6, pp. 893-901, 2007.

[12] W. Macdonald, P. Campbell, J. Fisher, and A. Wennerberg, "Variation in surface texture measurements," Journal of Biomedical Materials Research Part B, vol. 70, no. 2, pp. 262269, 2004.

[13] A. V. Lombardi Jr., K. R. Berend, and T. H. Mallory, "Hydroxyapatite-coated titanium porous plasma spray tapered stem: experience at 15 to 18 years," Clinical Orthopaedics and Related Research, vol. 453, pp. 81-85, 2006.

[14] K. Yoon, H. J. Kim, J. H. Lee, S. B. Kang, N. H. Seong, and K. H. Koo, "A randomized clinical trial of cementless femoral stems with and without hydroxyapatite/tricalciumphosphate coating: an 8- to 12-year follow-up study," Journal of Arthroplasty, vol. 22, no. 4, pp. 504-508, 2007.

[15] E. W. H. Bodde, R. S. Z. Kowalski, P. H. M. Spauwen, and J. A. Jansen, "No increased bone formation around alendronate or omeprazole loaded bioactive bone cements in a femoral defect," Tissue Engineering Part A, vol. 14, no. 1, pp. 29-39, 2008.

[16] R. G. G. Russell, "Bisphosphonates: from bench to bedside," Annals of the New York Academy of Sciences, vol. 1068, no. 1, pp. 367-401, 2006.

[17] T. Jakobsen, S. Kold, J. E. Bechtold, B. Elmengaard, and K. Søballe, "Effect of topical alendronate treatment on fixation of implants inserted with bone compaction," Clinical Orthopaedics and Related Research, vol. 444, pp. 229-234, 2006.

[18] T. Jakobsen, S. Kold, J. E. Bechtold, B. Elmengaard, and K. Søballe, "Local alendronate increases fixation of implants inserted with bone compaction: 12-week canine study," Journal of Orthopaedic Research, vol. 25, no. 4, pp. 432-441, 2007.

[19] K. McLeod, G. I. Anderson, N. K. Dutta, et al., "Adsorption of bisphosphonate onto hydroxyapatite using a novel co-precipitation technique for bone growth enhancement," Journal of Biomedical Materials Research Part A, vol. 79, no. 2, pp. 271-281, 2006.

[20] J. Horn, "The proton-pump inhibitors: similarities and differences," Clinical Therapeutics, vol. 22, no. 3, pp. 266-280, 2000.

[21] J. P. Mattsson, K. Vaananen, B. Wallmark, and P. Lorentzon, "Omeprazole and bafilomycin, two proton pump inhibitors: differentiation of their effects on gastric, kidney and bone H-translocating ATPases," Biochimica et Biophysica Acta, vol. 1065, no. 2, pp. 261-268, 1991.

[22] K. Mizunashi, Y. Furukawa, K. Katano, and K. Abe, "Effect of omeprazole, an inhibitor of $\mathrm{H}^{+}, \mathrm{K}^{+}$-ATPase, on bone resorption in humans," Calcified Tissue International, vol. 53, no. 1, pp. 21-25, 1993.

[23] A. V. Rousselle and D. Heymann, "Osteoclastic acidification pathways during bone resorption," Bone, vol. 30, no. 4, pp. 533-540, 2002.
[24] G. Vaes, "Cellular biology and biochemical mechanism of bone resorption. A review of recent developments on the formation, activation, and mode of action of osteoclasts," Clinical Orthopaedics and Related Research, no. 231, pp. 239271, 1988.

[25] M. Zaidi, "Modularity of osteoclast behaviour and "modelspecific" inhibition of osteoclast function," Bioscience Reports, vol. 10, no. 6, pp. 547-556, 1990.

[26] X. Li, Q. Cui, C. Kao, G. J. Wang, and G. Balian, "Lovastatin inhibits adipogenic and stimulates osteogenic differentiation by suppressing PPAR $\gamma 2$ and increasing Cbfa1/Runx 2 expression in bone marrow mesenchymal cell cultures," Bone, vol. 33, no. 4, pp. 652-659, 2003.

[27] G. E. Gutierrez, D. Lalka, I. R. Garrett, G. Rossini, and G. R. Mundy, "Transdermal application of lovastatin to rats causes profound increases in bone formation and plasma concentrations," Osteoporosis International, vol. 17, no. 7, pp. 1033-1042, 2006.

[28] G. Mundy, R. Garrett, S. Harris, et al., "Stimulation of bone formation in vitro and in rodents by statins," Science, vol. 286, no. 5446, pp. 1946-1949, 1999.

[29] G. E. Gutierrez, J. Zhang, S. Jadhav, M. A. Steve, and G. R. Mundy, "Enhanced expression of BMP2/Smad pathway and SP7 by statins during fracture repair," Bone, vol. 42, pp. S17S110, 2008.

[30] Q. O. Tang, G. T. Tran, Z. Gamie, et al., "Statins: under investigation for increasing bone mineral density and augmenting fracture healing," Expert Opinion on Investigational Drugs, vol. 17, no. 10, pp. 1435-1463, 2008.

[31] H. Uusitalo, J. Rantakokko, M. Ahonen, et al., "A metaphyseal defect model of the femur for studies of murine bone healing," Bone, vol. 28, no. 4, pp. 423-429, 2001.

[32] R. V. A. Baron and A. Silvergate, "Processing of undecalcified bone specimens for bone histomorphometry," in Bone Histomorphometry: Teachniques and Interpretation, pp. 13-35, CRC Press, Boca Raton, Fla, USA, 1983.

[33] C. Maniatopoulos, R. M. Pilliar, and D. C. Smith, “Threaded versus porous surfaced designs for implant stabilization in bone-endodontic implant model," Journal of Biomedical Materials Research, vol. 20, no. 9, pp. 1309-1333, 1986.

[34] C. Maniatopoulos, A. Rodriguez, D. A. Deporter, and A. H. Melcher, "An improved method for preparing histological sections of metallic implants," The International Journal of Oral \& Maxillofacial Implants, vol. 1, no. 1, pp. 31-37, 1986.

[35] C. Maniatopoulos and B. Tsatsas, "Endodontic implants. Clinical application of techniques," Odontostomatologike Proodos, vol. 40, no. 4, pp. 221-233, 1986.

[36] M. J. Rogers, "New insights into the molecular mechanisms of action of bisphosphonates," Current Pharmaceutical Design, vol. 9, no. 32, pp. 2643-2658, 2003.

[37] R. G. G. Russell, "Bisphosphonates: mode of action and pharmacology," Pediatrics, vol. 119, no. 2, pp. S150-S162, 2007.

[38] I. Mathov, L. I. Plotkin, C. L. Sgarlata, J. Leoni, and T. Bellido, "Extracellular signal-regulated kinases and calcium channels are involved in the proliferative effect of bisphosphonates on osteoblastic cells in vitro," Journal of Bone and Mineral Research, vol. 16, no. 11, pp. 2050-2056, 2001.

[39] F. von Knoch, C. Jaquiery, M. Kowalsky, et al., "Effects of bisphosphonates on proliferation and osteoblast differentiation of human bone marrow stromal cells," Biomaterials, vol. 26, no. 34, pp. 6941-6949, 2005. 
[40] G. I. Im, S. A. Qureshi, J. Kenney, H. E. Rubash, and A. S. Shanbhag, "Osteoblast proliferation and maturation by bisphosphonates," Biomaterials, vol. 25, no. 18, pp. 4105-4115, 2004.

[41] L. I. Plotkin, R. S. Weinstein, A. M. Parfitt, P. K. Roberson, S. C. Manolagas, and T. Bellido, "Prevention of osteocyte and osteoblast apoptosis by bisphosphonates and calcitonin," Journal of Clinical Investigation, vol. 104, no. 10, pp. 13631374, 1999.

[42] L. I. Plotkin, J. I. Aguirre, S. Kousteni, S. C. Manolagas, and T. Bellido, "Bisphosphonates and estrogens inhibit osteocyte apoptosis via distinct molecular mechanisms downstream of extracellular signal-regulated kinase activation," Journal of Biological Chemistry, vol. 280, no. 8, pp. 7317-7325, 2005.

[43] G. Duque and D. Rivas, "Alendronate has an anabolic effect on bone through the differentiation of mesenchymal stem cells," Journal of Bone and Mineral Research, vol. 22, no. 10, pp. 1603-1611, 2007.

[44] L. Fu, T. Tang, Y. Miao, S. Zhang, Z. Qu, and K. Dai, "Stimulation of osteogenic differentiation and inhibition of adipogenic differentiation in bone marrow stromal cells by alendronate via ERK and JNK activation," Bone, vol. 43, no. 1, pp. 40-47, 2008.

[45] N. Giuliani, M. Pedrazzoni, G. Negri, G. Passeri, M. Impicciatore, and G. Girasole, "Bisphosphonates stimulate formation of osteoblast precursors and mineralized nodules in murine and human bone marrow cultures in vitro and promote early osteoblastogenesis in young and aged mice in vivo," Bone, vol. 22, no. 5, pp. 455-461, 1998.

[46] Y. Xiong, H. J. Yang, J. Feng, Z. L. Shi, and L.-D. Wu, "Effects of alendronate on the proliferation and osteogenic differentiation of MG-63 cells," Journal of International Medical Research, vol. 37, no. 2, pp. 407-416, 2009.

[47] T. Fujita, N. Izumo, R. Fukuyama, et al., "Incadronate and etidronate accelerate phosphate-primed mineralization of MC4 cells via ERK1/2-Cbfal signaling pathway in a Rasindependent manner: further involvement of mevalonatepathway blockade for incadronate," Japanese Journal of Pharmacology, vol. 86, no. 1, pp. 86-96, 2001.

[48] J. Karrholm, B. Borssen, G. Lowenhielm, and F. Snorrason, "Does early micromotion of femoral stem prostheses matter? 4-7-year stereoradiographic follow-up of 84 cemented prostheses," Journal of Bone and Joint Surgery. British, vol. 76, no. 6, pp. 912-917, 1994.

[49] L. Chun, J. Yoon, Y. Song, P. Huie, D. Regula, and S. B. Goodman, "The characterization of macrophages and osteoclasts in tissues harvested from revised total hip prostheses," Journal of Biomedical Materials Research, vol. 48, no. 6, pp. 899-903, 1999.

[50] V. L. Fornasier, S. B. Goodman, K. Protzner, M. Kamel, Y. Song, and A. Shojaci, "The role of implant alignment on stability and particles on periprosthetic osteolysis-a rabbit model of implant failure," Journal of Biomedical Materials Research Part B, vol. 70, no. 2, pp. 179-186, 2004.

[51] S. Goodman, H. Saastamoinen, N. Shasha, and A. Gross, "Complications of ilioischial reconstruction rings in revision total hip arthroplasty," Journal of Arthroplasty, vol. 19, no. 4, pp. 436-446, 2004.

[52] J. Tuukkanen and H. K. Vaananen, "Omeprazole, a specific inhibitor of $\mathrm{H}^{+}-\mathrm{K}^{+}$-ATPase, inhibits bone resorption in vitro," Calcified Tissue International, vol. 38, no. 2, pp. 123-125, 1986.

[53] B. Peter, O. Gauthier, S. Laïb, et al., "Local delivery of bisphosphonate from coated orthopedic implants increases implants mechanical stability in osteoporotic rats," Journal of Biomedical Materials Research Part A, vol. 76, no. 1, pp. 133-143, 2006. 

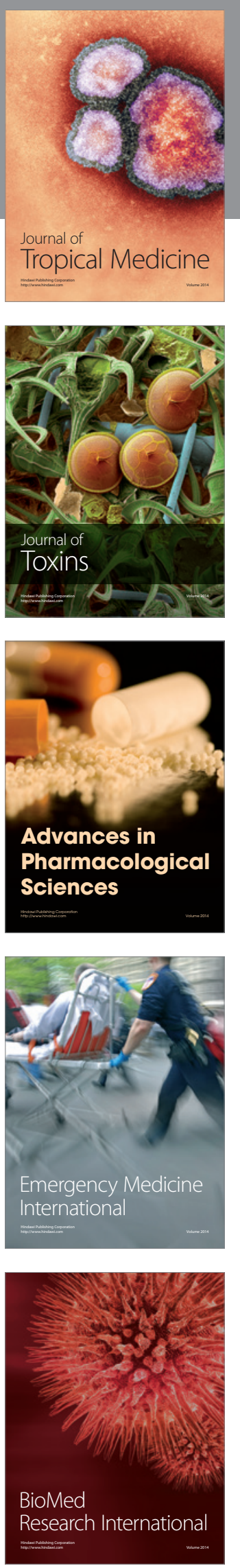


The Scientific World Journal
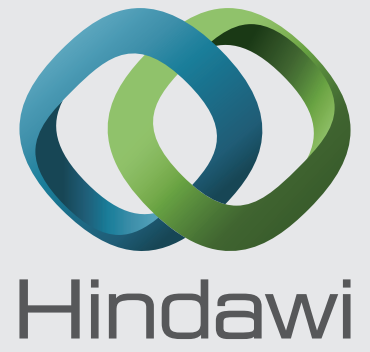

Submit your manuscripts at

http://www.hindawi.com
